\title{
A brief overview about laser-ablated molybdenum plasma properties
}

\begin{abstract}
First step in molybdenum isotope separation for medical purposes project, based in laser ablation followed by electromagnetic separation methods, is the characterization of ablated plasma plume. In this work, a depth profile emission spectroscopy study of laser-ablated molybdenum plasma expanding on air at atmospheric pressure was performed, considering Local Thermodynamic Equilibrium and one-dimensional uniform expanding models. Plasma temperature, electron density, electron population distribution and electron impact width parameter were evaluated along plasma expansion axis and reach average values of $15000 \mathrm{~K}, 1018 \mathrm{~cm}-3,1 \%$ of electron population at $4 \mathrm{~d} 45 \mathrm{~s} 6 \mathrm{~s}$ state and $(0.017 \pm 0.008) \mathrm{nm}$, respectively. Furthermore, mean velocity of $(5.0 \pm 0.7) \mathrm{km} / \mathrm{s}$ and the plasma lifetime that was $(160 \pm 14) \mathrm{ns}$ for length equal to $(1.80 \pm 0.05) \mathrm{mm}$ were measured. So, this work includes a description of mainly plasma plume parameters, which permits a discussion for isotope separation methods related to laser ablation.
\end{abstract}

Keywords: laser, plasma plum, wavelength, electronic population, atmospheric pressure, atoms
Volume I Issue 2 - 2017

\author{
Sternberg EMA,' Rodrigues NAS ${ }^{2}$ \\ 'Department of Physics, Federal Institute of Education, Brazil \\ ${ }^{2}$ Department of Physics, Aeronautics Institute of Technology, \\ Brazil
}

Correspondence: Emmanuela Sternberg, Department of Physics, Federal Institute of Education, Science and Technology of Espírito Santo, Federal University of Espírito Santo, Brazil, Tel (+55) 27 3246I600, Email emmanuela.sternberg@ifes.edu.br

Received: August 16, 2017| Published: September 08, 2017

\section{Introduction}

In Nuclear Medicine, the $99 \mathrm{~m}$ technetium isotope, ${ }^{99 \mathrm{~m}} \mathrm{Tc}$, is used as tracer in $\mathrm{CT}$ diagnosis exams, and the only source of ${ }^{99 \mathrm{~m}} \mathrm{Tc}$ is beta-decay of 99 molybdenum isotope, ${ }^{99} \mathrm{Mo}$. The problem is that a few aging reactors are working for attending world's needs of ${ }^{99} \mathrm{Mo}$, besides the fact that these processes involve highly enriched uranium (HEU). ${ }^{1-3}$ Intending to solve this problem, new methods for ${ }^{99} \mathrm{Mo}$ production are being proposed, such as neutron activation of ${ }^{98} \mathrm{Mo}$. To improve this method, it is suggested isotope separation methods to enhance the abundance of ${ }^{99} \mathrm{Mo}$ on the sample. A preliminary step to implement the suggested method is the generation and characterization of a laser-ablated plasma plume. Laser induced breakdown spectroscopy of molybdenum can determine expansion speed and lifetime ${ }^{4}$ after laser pulse of plasma jet, the portion of neutral and ionized species which constitutes the plasma, electronic population distribution, ${ }^{5}$ dynamics of plume expansion, ${ }^{4}$ electron density, temperature and electron impact width parameter. ${ }^{6}$ This work consists on laser ablation of molybdenum solid target and the characterization of the resulting plasma plume, aiming to analyze the possibility of application of isotope separation methods. So, the characterization of molybdenum by laser induced breakdown spectroscopy contributes not only for ${ }^{99} \mathrm{Mo}$ production but also with understanding fundamental concepts on laser-ablated plasmas.

\section{Experimental setup}

For molybdenum plasma plume generation, a Coherent AVIA 355-X Nd:YAG laser emitting pulses with $0.205 \mathrm{~mJ}$ energy and $25 \mathrm{~ns}$ duration (FWHM) in third harmonic $(355 \mathrm{~nm})$ was focused on a molybdenum solid sample with a laser fluence of $6 \mathrm{~J} / \mathrm{cm}^{2}$ and repetition rate equal to $1.2 \mathrm{kHz}$. The solid sample was placed on a XYtable, which movement is controlled by EMC2 software, intending to avoid sample cratering and plasma extinguishing. Figure 1 indicates a schematic experimental setup diagram. The plasma emitted light was collected by lenses set and focused on an optical fiber supported on a XYZ micrometer, which permits to execute a space-resolved study of plasma light distribution. Emitted light was analyzed through HR4000 and LIBS 2500+ spectrometers, both from Ocean Optics.

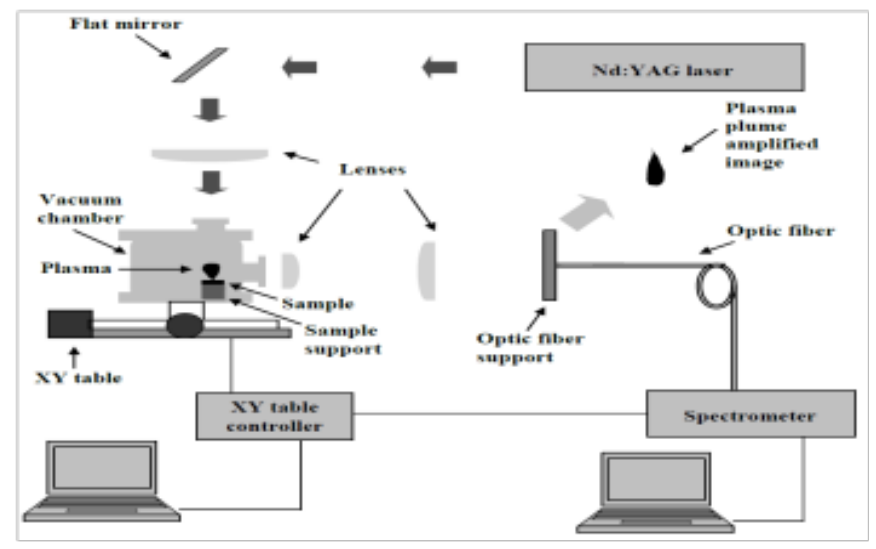

Figure I Experimental set-up. ${ }^{4}$

\section{Results and discussions}

The acquired spectra from plasma emitted light were investigated through direct comparison with theoretical spectra (Figure 2) constructed from NIST database for molybdenum neutral and singly ionized emission line. ${ }^{7}$ Based on assignment of emission lines for all emission wavelength range, it can be concluded that the light emitted from the plasma near the surface of the target is, mainly, due deexcitation of molybdenum ionized atoms and, as the plasma expands, it is due to transitions of neutral atoms to the ground state. Assigned experimental lines were used to determine electron temperature, electron density and electron population distribution, considering Local Thermodynamic Equilibrium Model. In this case, Saha equation 
(Equation 1) relates the electron density of the states with electron temperature for different ionization stages. ${ }^{9}$

$$
\frac{N_{e} N_{+}}{N_{0}}=2 \frac{g_{+}}{g_{0}}\left(\frac{2 \pi m_{e} k_{B} T}{h^{2}}\right)^{\frac{3}{2}} e^{\left\{\frac{-\left(E_{+}-E_{0}+E_{i}\right)}{k_{B} T}\right\}}
$$

In which $N$ is electron density of the state, $g$ is degeneracy, $m$ is electron mass, $E$ is energy of the level, $E$ is ionization energy. It is possible to establish a connection of emission line intensity and its electronic transition, so Equation 2 relates intensity $I$ and wavelength $\lambda$ of emission line with electron temperature and total electron density, where $A$ is spontaneous emission coefficient (and can be related to the probability of spontaneous emission), $k_{B}$ is Boltzmann's constant, $h$ is Planck's constant, and sub-indexes $\stackrel{B}{+}, 0$ and $e$ are related to the ionized atoms, neutral atoms and electrons, respectively.

$$
\frac{I_{+}}{I_{0}}=\frac{2}{N_{e}} \frac{\lambda_{0}}{\lambda_{+}} \frac{A_{+} g_{+}}{A_{0} g_{0}}\left(\frac{2 \pi m_{e} k_{B} T}{h^{2}}\right)^{\frac{3}{2}} e^{\left\{\frac{-\left(E_{+}-E_{0}+E_{i}\right)}{k_{B} T}\right\}}
$$

Electron temperature along plasma expansion axis was determined through Boltzmann plot (or its simplification called two lines method), when only information of emission lines related to transitions of atoms at the same ionization degree, and Saha-Boltzmann plot for the ratio of emission lines from different ionization degrees. So, electron temperature is inversely proportional to angular coefficient of linear fitting. Figure 3 presents a typical behavior of electron temperature, with a maximum value at the plume core, with mean value nearly $15000 \mathrm{~K}$. Replacing the calculated values for electron temperature in Equation 2, electron density $N_{e}$ was direct determined. A representative behavior of electron density along plasma expansion axis is depicted in Figure 4(A) and permits to evaluate that, at central portion of the plume, electron density reaches values around $10^{18}$ particles $/ \mathrm{cm}^{3}$. Once electron temperature values were measured, it also can be estimated a proportion of electron distribution population of each electronic level by means of Saha equation (Equation 1). Figure 4(B) reveals the time evolution of electron population of $4 \mathrm{~d}^{4} 5 \mathrm{~s} 6 \mathrm{~s}$ state, where, at the second moment, only spontaneous emission was taken into account.

Usually plasma electron density is determined by Stark broadening $\left({ }^{\Delta \lambda}\right.$ Stark $)$, which expression depends of electron impact width parameter $(\omega)$, according to Equation 3.

$$
{ }^{\Delta \lambda} \text { Stark }=2 \omega\left(\frac{N_{e}}{10^{16}}\right)
$$

However, database for molybdenum in literature is scarce, and this electron parameter is unknown. If it can be reputable that the experimental line broadening is mainly due Stark and instrumental broadening mechanisms fitted by lorentzian profile and, once electron density was determined by Saha-Boltzmann equation, Stark broadening expression permits to determine the electron impact width parameter. For $273.339 \mathrm{~nm}$ molybdenum plasma emission line expanding on air at atmospheric pressure and temperature nearly $8000 \mathrm{~K}$, the electron impact width parameter was measured equal to $(0.017 \pm 0.008) \mathrm{nm} .{ }^{6}$ Plasma expanding velocity can be determined as the ratio between length of laser scattered light equal to $(0.35 \pm 0.05)$ $\mathrm{mm}$ and laser pulse duration equal to $(75 \pm 5) \mathrm{ns}$, considering that the plasma expansion is uniform. So, the calculated plasma expansion velocity is $(5.0 \pm 0.7) \mathrm{km} / \mathrm{s}$ and, with measured plume length equal to $(1.80 \pm 0.05) \mathrm{mm}$, plasma lifetime is determined equal to $(160 \pm 14) \mathrm{ns}$. Table 1 summarizes molybdenum plasma properties of laser-ablated plume expanding on air at atmospheric pressure and it presents a comparison with reference data. ${ }^{8-12}$

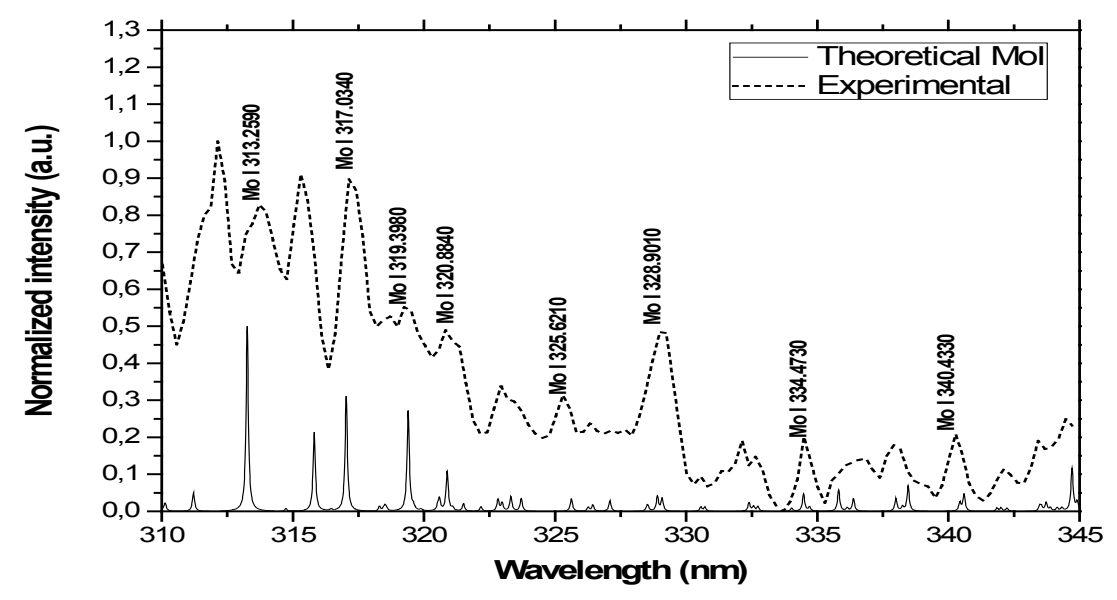

Figure 2 Assignment of the molybdenum emission lines for neutral atoms (Mo I).

Dashed line represents experimental data and solid line is theoretical spectrum. ${ }^{4}$ 


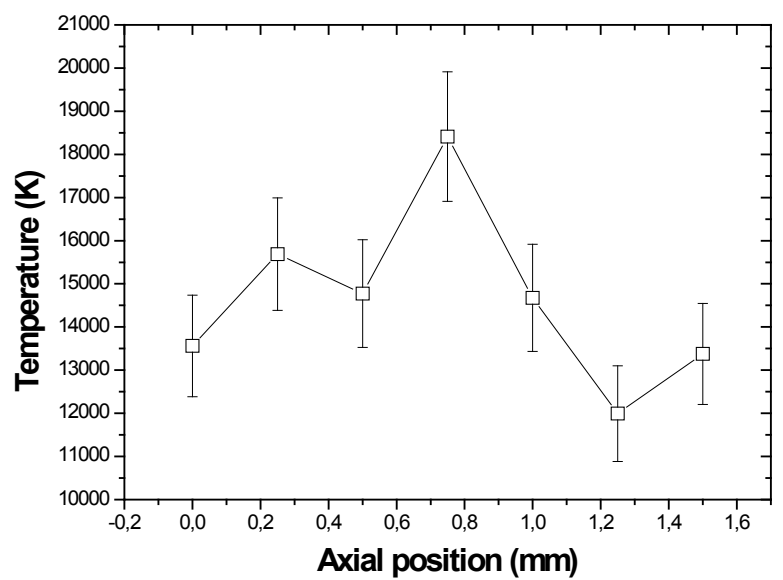

Figure 3 Electron temperature evolution along plume expansion axis. ${ }^{5}$
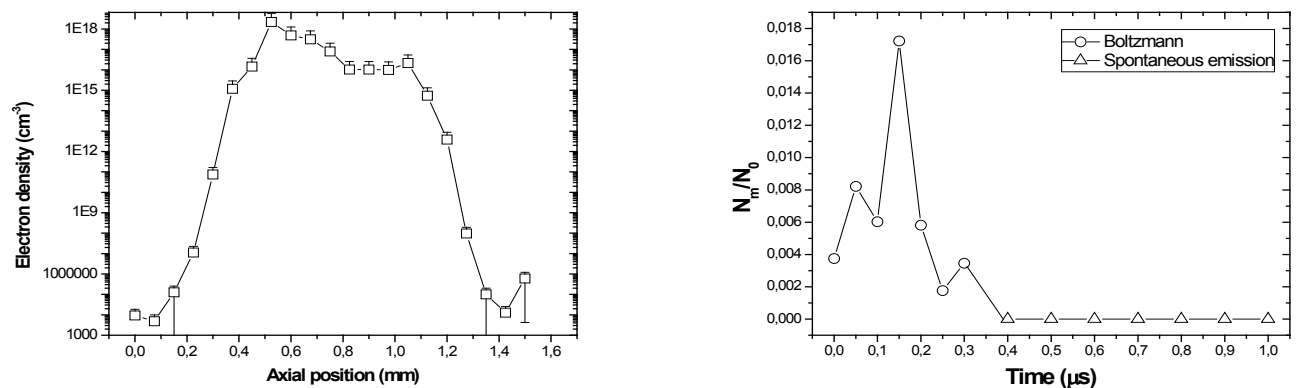

Figure 4 Electron density distribution. 4a Total electron density of plume along expansion axis, 6 and $4 \mathrm{~b}$ time evolution of electron population of $4 \mathrm{~d} 45 \mathrm{~s} 6 \mathrm{~s}$ state. 5

Table I Molybdenum plasma properties

\begin{tabular}{llll}
\hline & Atmospheric pressure & Mo references & References for other metals \\
\hline Expansion velocity & $5.0 \mathrm{~km} / \mathrm{s}$ & -- & $10.0 \mathrm{~km} / \mathrm{s}$ \\
Plume length & $1.8 \mathrm{~mm}$ & --- & $2.0 \mathrm{~mm}$ \\
Electron temperature & $15000 \mathrm{~K}$ & $10000 \mathrm{~K}$ & $10500 \mathrm{~K}-13000 \mathrm{~K}$ \\
Electron density & $2.2 \times 10^{18} \mathrm{~cm}^{-3}$ & $5.0 \times 10^{16} \mathrm{~cm}^{-3}$ & $10^{16} \mathrm{~cm}^{-3}-10^{18} \mathrm{~cm}^{-3}$ \\
Electron impact width parameter & $0.017 \mathrm{~nm}$ & --- & $0.015 \mathrm{~nm}$ \\
\hline
\end{tabular}

\section{Conclusion}

Emission spectroscopy study of laser-ablated Mo plasma along plume expansion axis was performed. Plasma parameters as plasma expansion speed value around $5.0 \mathrm{~km} / \mathrm{s}$ and plasma lifetime nearly 150ns was calculated using uniform and one-dimensional expanding model. Due direct comparison between experimental and theoretical spectra and emission lines assignment, it was demonstrated that the plume evolves from the atoms mainly at ionized states, near target surface, to the atoms at ground state at the end of plume emission, so that the plasma becomes a neutral, monatomic and at ground state Mo jet. Considering Local Thermodynamic Equilibrium Model and SahaBoltzmann equation, electron temperature was calculated around $15000 \mathrm{~K}$, reaches its maximum value at central portion of the plume and decays at the boundaries. Also, electron density reaches values of $10^{18} \mathrm{~cm}^{-3}$ and electron impact width parameter was determined by Stark broadening expression equal to $(0.017 \pm 0.008) \mathrm{nm}$ for temperature of $8000 \mathrm{~K}$. The obtained results reveal laser ablation as an auxiliary method for isotope separation methods and that laserablated Mo plasma plume could be used for ${ }^{99}$ Mo production.

\section{Acknowledgments}

None.

\section{Conflicts of interest}

The auhtor declares there is no conflict of interest. 


\section{References}

1. Soonawala D, Amin T, Ebmeier KP, et al. Statistical Parametric Mapping of 99mTc-HMPAO-SPECT Images for the Diagnosis of Alzheimer's Disease: Normalizing to Cerebellar Tracer Uptake. NeuroImage. 2002;17(3):1193-2002.

2. Verbeek P. Report on molybdenum-99 production for nuclear medicine2010-2020. Association of Imaging Producers \& Equipment Suppliers. 2008 .

3. Matos JB, Oliveira ML, Sternberg EMA, et al. Generation of an Atomic Beam by Using Laser Ablation for Isotope Separation Purposes. Journal of Aerospace Technology and Management. 2012;4(4):413-420.

4. Sternberg EMA, Rodrigues NAS, Sbampato ME, et al. Excited states time evolution on a laser-ablated molybdenum plume. Applied Physics B. 2014;116(4):985-989.

5. Sternberg EMA, Rodrigues NAS, Amorim J, et al. Plasma properties of $355 \mathrm{~nm}$ and $532 \mathrm{~nm}$ laser-ablated molybdenum target at atmospheric pressure. Journal of Physics: Conference Series. 2012;370(1):1-8.

6. Sternberg EMA, Rodrigues NAS, Amorim J. Molybdenum electron impact width parameter measurement by laser induced breakdown spectroscopy. Applied Physics B. 2016;122(21).
7. http://www.nist.gov/physlab/data/asd.cfm accessed at 21/11/2012

8. Jakubowska K, Kubkowska M, Blagoev A, et al. A spectroscopic study of laser ablation plasma from Mo target. Physica Scripta. 2014.

9. Hanif M, Salik M. Optical emission studies of molybdenum plasma produced by an Nd: YAG laser. Journal of Russian Laser Research. 2014;35(3):230-238.

10. Farid N, Wang H, Li C, et al. Effect of background gases at reduced pressures on the laser treated surface morphology, spectral emission and characteristics parameters of laser produced Mo plasmas. Journal of Nuclear Materials. 2014;438(1-3):183-189.

11. Farid N, Harilal SS, Ding H, et al. Emission features and expansion dynamics of nanosecond laser ablation plumes at different ambient pressures. Journal of Applied Physics. 2014;115(3).

12. Lide DR. CRC Handbook of Chemistry and Physics $87^{\text {th }}$ edn. CRC Press, Boca Raton, USA. 2006. 